\title{
Mouse models of UV-induced melanoma: genetics, pathology, and clinical relevance
}

\author{
Chi-Ping Day ${ }^{1,3}$, Rachel Marchalik ${ }^{1,3}$, Glenn Merlino ${ }^{1}$ and Helen Michael ${ }^{1,2}$
}

Melanocytes, a neural crest cell derivative, produce pigment to protect keratinocytes from ultraviolet radiation (UVR). Although melanocytic lesions such as nevi and cutaneous malignant melanomas are known to be associated with sun exposure, the role of UVR in oncogenesis is complex and has yet to be clearly elucidated. UVR appears to have a direct mutational role in inducing or promoting melanoma formation as well as an indirect role through microenvironmental changes. Recent advances in the modeling of human melanoma in animals have built platforms upon which prospective studies can begin to investigate these questions. This review will focus exclusively on genetically engineered mouse models of UVR-induced melanoma. The role that UVR has in mouse models depends on multiple factors, including the waveband, timing, and dose of UVR, as well as the nature of the oncogenic agent(s) driving melanomagenesis in the model. Work in the field has examined the role of neonatal and adult UVR, interactions between UVR and common melanoma oncogenes, the role of sunscreen in preventing melanoma, and the effect of UVR on immune function within the skin. Here we describe relevant mouse models and discuss how these models can best be translated to the study of human skin and cutaneous melanoma.

Laboratory Investigation (2017) 97, 698-705; doi:10.1038/labinvest.2016.155; published online 16 January 2017

\section{INTRODUCTION}

Melanocytes are the pigment-producing cells of the body and reside in a variety of anatomic locations, including skin, eyes, inner ear, and mucosal surfaces. In the human skin, the primary function of melanocytes is protection from ultraviolet radiation (UVR), which is considered to be a complete carcinogen as it can function as both a tumor initiator and promoter. Melanocytes produce melanin pigment and transfer it to adjacent keratinocytes packaged in melanosomes, which use the pigment to protect their nuclei from UVR damage. Melanocytic lesions, benign nevi or malignant melanomas, have been associated with UVR exposure, although both can occur in non-UVR-exposed sites, including mucosal surfaces.

Although UVR exposure is believed to have an important role in the development of nevi and cutaneous melanomas, the mechanisms remain unclear. Epidemiological studies have linked childhood sunburn, intermittent sunburn, and tanning (through both outdoors and in tanning beds) to increased melanoma risk. ${ }^{1-5}$ People with red hair and non-tanning pale skin have a higher risk of melanoma owing to higher production of pheomelanin, which is less protective against UVR than eumelanin. ${ }^{6}$ Melanoma incidence in susceptible people is higher in geographical regions with higher UVR indices. ${ }^{7}$

Sunlight is overwhelmingly the greatest source of UVR exposure reaching the earth and is composed of 95\% UVA $(320-340 \mathrm{~nm})$ and 5\% UVB (290-320 nm). UVC rays are blocked by stratospheric ozone. ${ }^{8,9}$ UVA penetrates more deeply into the skin and up to $50 \%$ of solar UVA can reach junctional melanocytes compared with only $9-15 \%$ of UVB (Figure 1). Many indoor tanning beds use high-dose UVA with little-to-no UVB. ${ }^{10}$ Both UVA and UVB can cause DNA damage and alter the microenvironment within the skin. ${ }^{11-13}$ Epidemiological data suggest potential roles for both UVA and UVB in melanoma development, but the comparative importance of UVA and UVB on human melanoma development remains controversial.

Much of the attention on UVR and melanoma has focused on the mutagenic role of UVR. In vitro studies show that UVB causes the formation of 6-4 photoproducts and signature $\mathrm{CC}>\mathrm{TT}$ mutations at dipyrimidine sites through the

\footnotetext{
'Laboratory of Cancer Biology and Genetics, Center for Cancer Research, National Cancer Institute, NIH, Bethesda, MD, USA and ${ }^{2}$ Department of Veterinary Medicine, University of Maryland, College Park, MD, USA

Correspondence: G Merlino, PhD or Dr H Michael, DVM, Laboratory of Cancer Biology and Genetics, National Cancer Institute, NIH, 37 Convent Drive, MSC 4264, Building 37, Room 5002, Bethesda, MD 20892, USA.

E-mail: gmerlino@helix.nih.gov or helen.michael@nih.gov

${ }^{3}$ These authors contributed equally to this work.

Received 31 October 2016; revised 14 December 2016; accepted 15 December 2016
} 

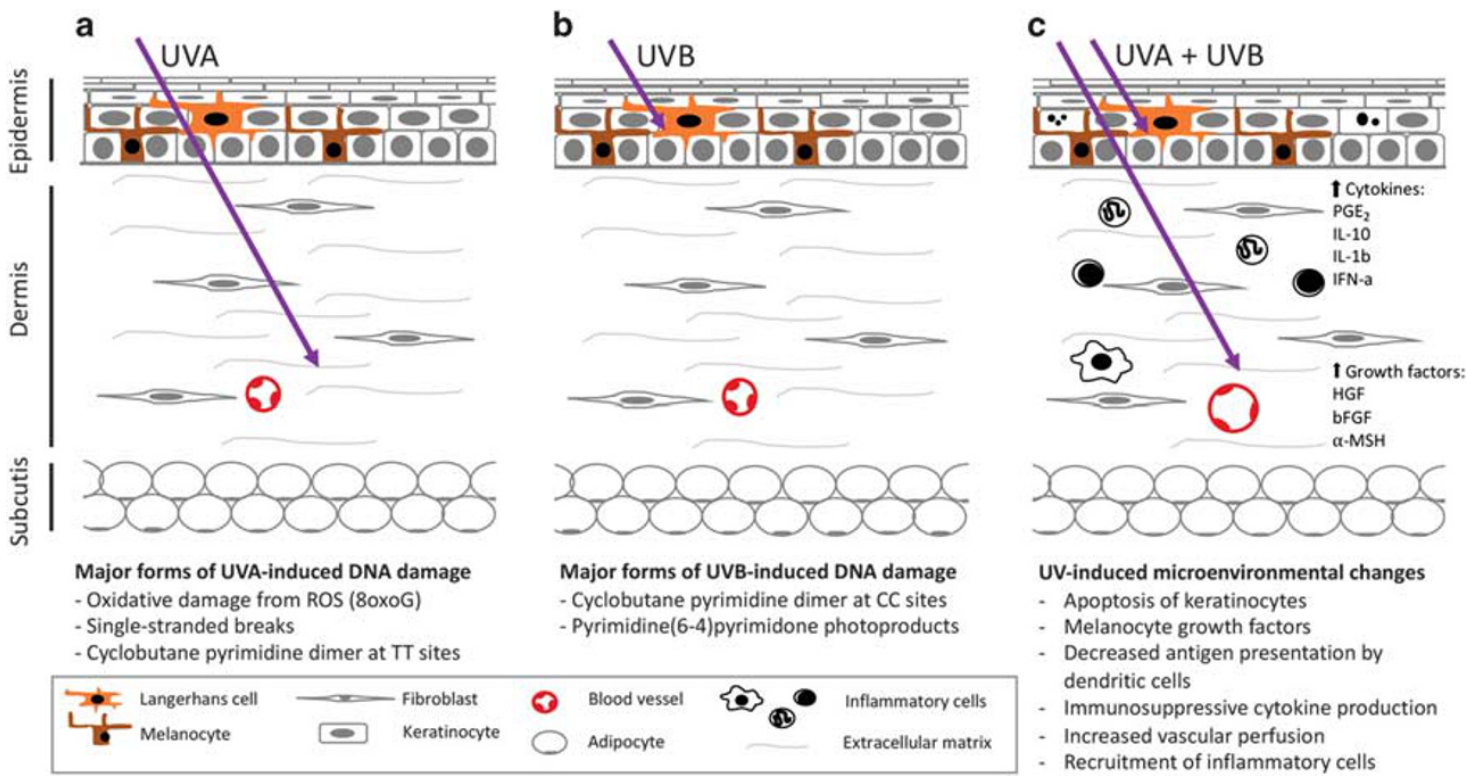

Figure 1 Major effects of UVR on the skin. UVA (a) and UVB (b) penetrate into the skin different amounts (purple arrow) and cause different types of DNA damage. In addition to DNA damage, UVA/UVB exposure causes a wide variety of skin microenvironmental changes (c).

formation of cyclobutane pyrimidine dimers (CPDs), although other patterns, including $\mathrm{C}>\mathrm{T}$ transitions at dipyrimidine sites, are also seen in vitro (Figure 1b). ${ }^{14}$ UVA is a less potent DNA-damaging agent and can damage DNA indirectly through oxidative damage from reactive oxygen species and through formation of CPDs at TT sites (Figure 1a), which usually do not lead to $\mathrm{C}>\mathrm{T}$ transitions. ${ }^{15-17}$ Human cutaneous melanomas are highly mutated with a predominance of $\mathrm{C}>\mathrm{T}$ transitions at dipyrimidine sites but fewer CC $>$ TT transitions, which is consistent with UVR mutagenesis. Genomic studies have identified important oncogenes (especially BRAF and NRAS) and tumor suppressors (eg, CDKN2A and NF1) involved in melanoma that have advanced our understanding of different melanoma subtypes and therapeutics. Readers who are interested in these findings should refer to some of the excellent reviews available on the subject. ${ }^{18,19}$ Interestingly, the driver mutations in the RAS/RAF pathway, including $\mathrm{BRAF}^{\mathrm{V} 600 \mathrm{E}}$, present in $60-90 \%$ of nevi and approximately $50 \%$ of melanomas, are not UVR-type mutations. ${ }^{20-24}$ These non-canonical mutations may or may not be related to UVR; in vitro studies have found that up to $10 \%$ of UVR-induced mutations can be $\mathrm{T}>\mathrm{A}$ transversions. ${ }^{14}$ Regardless, BRAF mutations are most common in sun-exposed nevi and melanoma, suggesting a link to UVR. ${ }^{20,25,26}$ BRAF mutations may well represent the initiation event for the formation of melanocytic nevi. ${ }^{27,28}$

In addition to mutagenesis, effects of UV on the skin microenvironment likely also have a role in the initiation and progression of melanoma (Figure 1c). Within the epidermis, each melanocyte is in contact with 30-40 neighboring keratinocytes through long dendritic processes. ${ }^{29}$ Keratinocytes and dermal fibroblasts produce many melanocyte growth factors, including basic fibroblast growth factor, hepatocyte growth factor (HGF), and endothelin 1 and $3 .^{30}$ Crosstalk between keratinocytes and melanocytes, and other cells within the skin microenvironment, allows adaption to external stimuli. Following UVR, surviving keratinocytes secrete melanocyte growth factors (including $\alpha-\mathrm{MSH}, \mathrm{EDN} 1)$, which increase cytokine and melanin production. ${ }^{29,31}$ In addition, direct and indirect interactions between melanoma cells and keratinocytes and fibroblasts can influence the behavior and metastatic potential of melanoma cells. ${ }^{32-35}$ There is increasing evidence that UVR-induced microenvironmental changes may be important during initiation and progression of melanocytic lesions.

The long latency between childhood and early adulthood sun exposure and melanoma formation makes it especially challenging to prospectively study the role of UVR on melanoma development. As UVR can function in multiple capacities as tumor initiator, tumor promoter and inducer of microenvironmental changes, the most significant roles for UVR can be difficult to identify by studying human melanoma patients alone.

Animal models, on the other hand, provide a platform for prospective studies to investigate specific questions and address causative associations. Although several species have been involved in the modeling of UVR-induced cutaneous melanoma, including opossum (Monodelphis domestica), platyfish (Xiphophorus spp.), and zebrafish (Danio rerio), in this review we will focus exclusively on genetically engineered mouse (GEM) models. ${ }^{36-40}$ The development of mouse models for melanoma was initially difficult owing to the resistance of wild-type mice to melanomagenesis, even after repeated exposure to chemical carcinogen, UVR, or both. ${ }^{41,42}$ However, use of hairless (Skh-hr2) mice treated with DMBA 
Table 1 Summary of UVR dosing and timing in selected mouse studies exploring the role of UVR in the development or progression of cutaneous melanoma

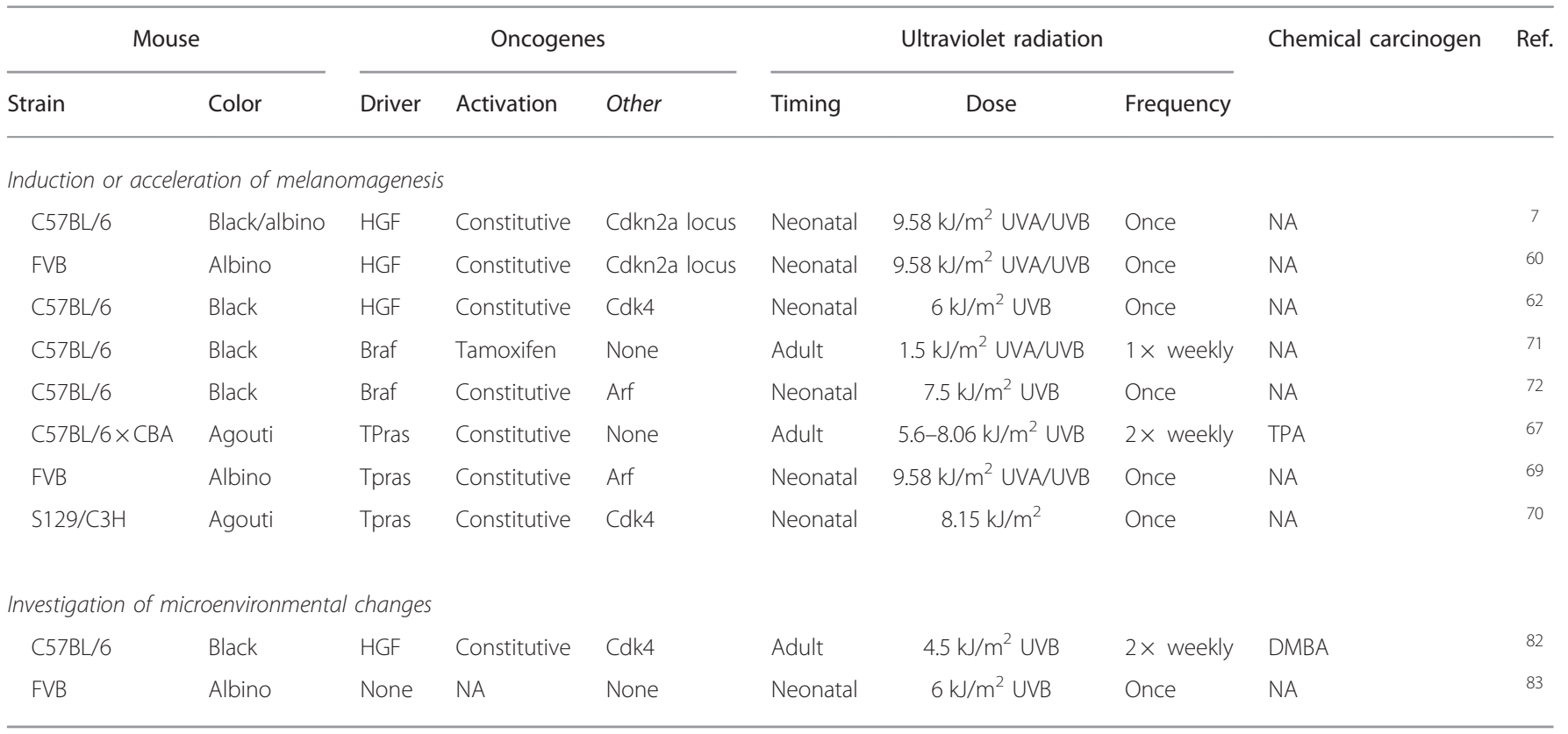

The first and second section of the table lists mouse models studies that have explored the effect of UVR on melanoma development and tumor microenvironment, respectively. In each section, the settings of the models (strain, coat color, and driver mutations) and experimental conditions (UVR timing, dose, and frequency, and whether or not a chemical carcinogen was administered to the mouse to enhance the mutagenic effect) are listed.

followed by repeated UVR was able to induce nevi and melanomas in treated animals. ${ }^{4-45}$ Interestingly, some of the melanomas and nevi in these mice had NRAS mutations near the sixty-first codon, which is a hotspot for human congenital nevi and melanomas. ${ }^{44}$ In the past decade, technological advances have generated GEM models that harbor oncogenic mutations associated with human melanoma to prospectively investigate clinically relevant questions about the role of UVR in cutaneous melanoma.

Differences in mouse and human skin need to be considered when assessing mouse melanoma studies that have investigated the role of UVR in melanoma biology. These studies use a variety of strains of mice, doses, timing and types of UVR administration (Table 1). Studies focus primarily on the ability of UVR to induce melanoma with or without a preexisting melanocytic lesion and on how UVRinduced microenvironmental changes can alter the progression of melanoma. Although the variability in the studies makes it difficult to directly compare results, it also highlights a wide variety of possible roles of UVR, depending on the dose, waveband, and context in the development and progression of cutaneous melanoma.

\section{SIMILARITIES AND DIFFERENCES BETWEEN MOUSE AND HUMAN SKIN}

Human and mouse skin have some distinct features. Both mouse and human skins are composed of dermis and epidermis, contain hair follicles, and have a similar distribution of blood vessels and nerves. Human skin is thicker $(1.5-6 \mathrm{~mm})$, with more epidermal layers $(5-8)$ and rete ridges, and is adherent to underlying tissues. In contrast, mouse skin is thinner $(0.7-0.8 \mathrm{~mm})$ with fewer epidermal keratinocyte layers (2-3), has no rete ridges, does not adhere to underlying tissue, and has a panniculus muscle layer. Mice also have a heavy hair coat that protects their skin from UVR. In the human skin, melanocytes are located along the dermalepidermal junction as well as in hair follicles. In the mouse skin, the vast majority of melanocytes are restricted to the hair follicles. During embryonic development in both species, melanoblasts migrate out of the neural tube, through the dermis to the dermal-epidermal junction. As hair follicles develop, some of these junctional melanocytes move into the developing follicles. In wild-type mice, junctional melanocytes do not persist after the neonatal stage in haired areas. As junctional melanocytes are not present in wild-type mice, melanomas derived from mouse models may arise from the cells of origin that differ from human melanomas. Additionally, these differences in anatomical location mean that in wild-type mice melanocytes are not interacting directly with keratinocytes and are receiving very different microenvironmental signals compared with melanocytes in the human epidermis.

Although melanocytes in both species produce both eumelanin and pheomelanin, these are produced in different frequencies. ${ }^{46,47}$ UVR increases the quantity of melanin produced and can alter the type produced. ${ }^{48}$ In humans, 
the frequency of each type of melanin varies widely with skin and hair coloration: people with darker skin produce higher quantities of eumelanin, while fair-skinned red heads produce a higher proportion of pheomelanin. Mice generally produce both types of melanin as well, but in many cases mouse strains produce either primarily eumelanin or pheomelanin. ${ }^{46}$

Human and mouse skin microenvironments also have some notable differences. Because of the dense hair coat, mouse skin undergoes frequent hair cycles. In each cycle, the hair follicles grow and degenerate, leading to turnover of mature melanocytes and cycling of melanocyte stem cells in the bulge of the hair follicle. Hair follicle involution also involves activation of immune cells, including macrophages and $\mathrm{T}$ cells in the skin. ${ }^{49}$ In contrast, human skin does not exhibit obvious hair cycles. Finally, the skin microbiome and resident immune cells are different between the two species. ${ }^{50}$ These microenvironmental changes may have implications both in the development of cutaneous melanoma and in the immunological responses in human and mouse skin.

In summary, mouse and human skin have some anatomical and functional differences that influence their response to UVR and the development of melanoma. The contrasting melanocyte distribution and resultant differences in melanocyte microenvironment in the human and mouse skin may be a major factor in the different susceptibility to melanoma in these species. These differences should be considered when choosing mouse models of UVR-induced melanoma and in translating findings to human melanoma patients. Careful choice of the appropriate model and experimental planning will provide the most valuable information for translational studies.

\section{MODELS EXPLORING THE ROLE OF UVR IN INDUCING OR ACCELERATING MELANOMA FORMATION}

\section{Neonatal UVR Exposure can Induce Melanoma in Mice} Without a Dominant Driver Mutation

A number of GEM models have been recruited to explore the role of UVR melanomagenesis, including those overexpressing HGF, the ligand for the receptor tyrosine kinase MET. HGF/MET signaling has a significant role in embryonic cell migration and has been associated with increased metastasis and drug resistance in human melanomas. ${ }^{51-54}$ Studies have shown that HGF promotes the survival of melanoblasts derived from neural crest and melanocytes in mouse skin. ${ }^{55}$ Human keratinocytes and skin fibroblasts secrete increased amounts of HGF following UVR exposure. ${ }^{56}$ Mouse skin expresses HGF in small amounts. ${ }^{57}$ Mice broadly expressing an HGF transgene gain constitutive activation of pathways downstream of MET, resulting in increased skin pigmentation owing to survival of melanocytes at the epidermal-dermal junction, which persists into adulthood. ${ }^{58-60}$ Therefore, HGF-tg mice are considered to exhibit a 'humanized' melanocyte distribution in the skin.

HGF is not a strong driver in cutaneous melanoma. Without carcinogenic treatment (UVR or DMBA), melanomas occur rarely in the skin of HGF-tg mice within 1-2 years. ${ }^{60,61}$ Adult UVR, even at prolonged repetitive dosing, does not induce melanomas in HGF mice but can induce other skin tumors. ${ }^{60}$ In contrast, a single burning UVR dose at 3.5 days can induce nevi and melanoma in HGF-tg mice. ${ }^{61}$ This is relevant because childhood sunburn is recognized as an important risk factor for melanoma formation. ${ }^{1}$ Melanocytic nevi and melanomas arising in the HGF-tg model are histologically heterogeneous and recapitulate the variety seen in human cutaneous melanocytic lesions. Disruptions of the CDKN2A locus or activation of CDK4 accelerate melanoma development following neonatal UVR in the HGF model. ${ }^{62,63}$ Targeted expression of survivin can also enhance UV-induced melanogenesis by preventing apoptosis of UVR-exposed melanocytes, suggesting that the dysregulation of senescence or apoptosis can have critical roles in the transformation of melanocytes following neonatal UVR. ${ }^{64}$

Melanocytic cells undergo significant changes associated with differentiation between the end of gestation and by 1 week after birth. ${ }^{65}$ Therefore, neonatal UVR exposure is likely to affect a distinct population of melanocytic cells compared with adult UVR - namely, progenitor melanoblasts $v s$ mature melanocytes, respectively. This may help account for the increased risk of melanoma seen with childhood sunburn $v s$ adult sunburn in human patients. The UVRtreated HGF-tg mouse represents a good model for human melanomas that does not possess either a BRAF or NRAS mutation (approximately $30 \%$ of cutaneous melanomas). As there is no preexistent dominant driver oncogene, UVR may serve as both tumor initiator and promoter for 'de novo' melanomagenesis in the HGF-tg model. This feature makes the HGF-tg mouse especially suitable in evaluating etiological factors, environmental risks, and prevention of UVR-induced nevi and melanoma following childhood sun exposure.

\section{UVR can Enhance Melanomagenesis Following Activation of Dominant Driver Mutations}

In contrast to the use of neonatal UVR in the HGF-tg mouse models, studies examining the role of UVR in melanocytic nevi and melanomas with a dominant oncogenic driver mutation have primarily focused on adult UVR exposure. Mice with mutations in the RAS/RAF pathways (HRAS, NRAS, or BRAF) show melanocytic hyperplasia and increased numbers of extrafollicular melanocytes within the dermis compared with wild-type mice. These models are consistent with the theory that RAS/RAF mutations are founding mutations in melanocytic nevi and melanomas. ${ }^{27,28}$ According to this theory, subsequent UVR may lead to contributory mutations and/or microenvironmental changes that can promote melanomagenesis.

The first model to investigate the role of UVR in melanoma development expressed a powerful viral oncogene SV40 T antigen (TAg-tg) using a mouse tyrosinase promoter. ${ }^{66} \mathrm{TAg}$ tg mouse pups were exposed to daily UVR for 3-10 days, ${ }^{66}$ 
leading to melanomas. Following transplantation, some of the UVR melanomas became metastatic. This study demonstrated that UVR could promote tumor development in the context of a powerful driver oncogene. Based on this model, researchers then tried to build melanoma models by adding different combinations of altered alleles that are more relevant to human melanoma.

RAS mutations (primarily NRAS but occasionally HRAS) are common in human congenital nevi and cutaneous melanoma, and Chin et al ${ }^{67}$ observed that mice expressing a mutant HRAS transgene by virtue of the mouse tyrosinase promoter (TPras) and loss of p16 ${ }^{\text {Ink } 4 \mathrm{a}}$ developed melanoma without additional carcinogens. TPras mutant mice with wild-type $\mathrm{p} 16^{\text {Ink4a }}$ were used to compare the effects of a single exposure to the chemical carcinogen (DMBA) with twice weekly UVR and a known tumor promoter (TPA). ${ }^{68}$ DMBA was very efficient and induced melanocytic lesions with a high penetrance (86-88\%). UVB exposure alone induced melanocytic lesions only in $20 \%$ of mice and most of these lesions were nevi. Combined treatment with UVB and TPA increased the lesion penetrance to $78 \%$. Interestingly, the melanomas that arose in the UVR-exposed groups occurred only in albino TPras mice, suggesting a protective role for melanin in this model.

Other studies using TPras mice have investigated the role of neonatal UVR and cell cycle checkpoints on the development of RAS mutant melanomas. Kannan et $a l^{69}$ found that UVR exposure accelerated melanomagenesis in p19 Arf knockout mice but not in $\mathrm{p} 16^{\text {Ink4a }}$ knockout albino mice. In $\mathrm{p} 19^{\text {Arf }}$ knockout mice, UVR-induced melanomas demonstrated increased cdk6 expression, leading to disruption of the RB pathway. Hacker et $a l^{70}$ reported that neonatal UVR exposure also increased the frequency and accelerated the development of melanomas in TPras mice carrying a Cdk $4^{\mathrm{R} 24 \mathrm{C}}$ mutation. ${ }^{70}$ It is not clear whether these findings are specific to neonatal UVR or would also be found following adult UVR in these strains. These studies indicate that functional disruption of the $\mathrm{p} 16^{\text {Ink4a }} / \mathrm{CDK} 4 / \mathrm{CDK} 6 / \mathrm{RB}$ pathway can facilitate mutant RAS-driven melanomagenesis in UVR-associated and non UVR-associated pathways.

BRAF $^{\mathrm{V} 600 \mathrm{E}}$ mutations are the most common driver mutations in human cutaneous melanoma. Epidemiological and genomic data suggest that $\mathrm{BRAF}^{\mathrm{V} 600 \mathrm{E}}$ mutations may be the initiating lesion in melanocytic nevi, with subsequent UVR acting as a tumor promoter. To test this hypothesis, Viros et $a l^{71}$ induced BRAF ${ }^{\mathrm{V} 600 \mathrm{E}}$ mutations using tyrosinasedriven Cre-Lox recombination in 2-month-old mice followed by weekly broad-spectrum UVR starting at 3 months. This UVR regimen both accelerated melanoma formation in the BRAF model and increased the number of tumors per mouse. UVR-exposed tumors also had significantly higher numbers of somatic single-nucleotide variants and a higher percentage of $\mathrm{C}>\mathrm{T}$ transitions than non-UVR-exposed tumors, consistent with human melanomas and UVR mutagenesis. Exclusively in the UVR-exposed tumors, Trp53 mutations were additionally identified. $\operatorname{Trp53}$ is known to be a mutational target of UVR, and the $\mathrm{p} 19^{\mathrm{Arf}} / \mathrm{p} 53$ pathway facilitates BRAF $^{\mathrm{V} 600 \mathrm{E}}$ mutant mouse melanoma. ${ }^{71,72}$ These data also suggest that UVR following BRAF mutation can enhance the likelihood of malignant transformation of nevi.

\section{Melanin Pigment is Important for UVA Melanomagenesis} Prospective mouse modeling of UVA and UVB administration have provided valuable information about their role in melanomagenesis. Overall, in mice UVB is a stronger inducer of melanoma, provoking tumors in a pigment-independent manner and leading to more tumors and earlier tumor formation. ${ }^{15,73}$ UVA induction of melanoma, however, is dependent on pigment. UVA only induced oxidative damage in the presence of melanin, while UVB induced CPDs with equal frequency with or without melanin. ${ }^{15}$ The type of melanin may also impact the development of oxidative damage. Human epidemiological data and in vitro experiments further support the role of pigment (particularly pheomelanin) in enhancing UVA-induced DNA damage. ${ }^{74-77}$ Studies using mice with varying amounts of pheomelanin show that increased oxidative damage in melanocytes with high levels of pheomelanin can occur independent of UVR, indicating the need for further investigation of UVR and pigment. ${ }^{6}$ The type of melanin may impact the complex interactions between UVA and pigment. As the ratio of eumelanin and pheomelanin is different in mice and humans, translation of pigment studies to human disease is not straightforward. Attention should be paid to pigmentation and the type of UVR when planning or interpreting data from mouse UVR melanoma models. These studies support the notion that UVA and UVB likely both contribute to the formation of cutaneous melanoma. The differences in the types of mutations induced within the tumors by the two wavebands may have clinical implications. In any event, minimizing exposure to both UVA and UVB is important for preventing melanoma.

\section{Sunscreen Provides Partial Protection Against UVR- Induced DNA Damage and Melanoma}

Melanoma incidence has been rising over the past few decades, and there is a need for evidence-based advice for protecting against melanoma and other skin cancers. Sunscreen use appears both epidemiologically and experimentally to protect against melanoma, which further highlights the importance of UVR in melanomagenesis. Prospective mouse studies have shown that sunscreen use reduces, but does not eliminate, the risk of melanoma following neonatal or adult UVR. ${ }^{71,78,79}$ Sunscreen reduced clinical and histological evidence of UVR damage in the skin, including inflammation, skin darkening, and evidence of UVR damage to keratinocytes. ${ }^{71,79}$ DNA damage to melanocytes was also reduced, with reduced numbers of TT dimers and reduced numbers of $\mathrm{C}>\mathrm{T}$ transitions in mice treated with sunscreen before UVR exposure compared with 
controls. ${ }^{71,78}$ Although melanoma formation was reduced in sunscreen-protected mice, it was still higher than in non-UVR exposed mice. Sun-protective fabrics were superior in preventing UVR-melanoma than sunscreens. ${ }^{71}$ These studies all found that sunscreen use prevented melanoma despite using a variety of UVR doses, sunscreens, and mouse strains. ${ }^{71,78,79}$ These data provide strong, prospective support for the use of sunscreens to reduce melanomagenesis. However, sunscreen use does not entirely eliminate UVRinduced melanoma formation and prevention of sunburn alone does not remove the risk of melanoma. Mouse models of UVR-induced melanoma provide a powerful tool to test the efficacy of sunscreens and other preventive strategies in preventing melanoma and other UVR-induced skin damage.

\section{MODELS EXPLORING THE ROLE OF UVR-INDUCED MICROENVIRONMENTAL CHANGES IN MELANOMA FORMATION}

In addition to direct mutagenic effects, UVR can influence the microenvironment in ways that can enhance melanomagenesis. UVR has long been known to cause immunosuppression within the skin. UVR leads to locally decreased antigen presentation, expansion of regulatory $\mathrm{T}$ cells, and lower numbers of effector $\mathrm{T}$ cell in the exposed skin. ${ }^{80}$ In fact, the immunosuppressive effect of UVR is used to treat psoriasis and can suppress contact hypersensitivity. ${ }^{81}$ Therefore, several models have been developed to study how UVR-induced changes in the skin microenvironment influence melanoma development and progression.

Bald et a $l^{82}$ used the HGF-tg/Cdk $4^{\mathrm{R} 24 \mathrm{C}}$ mouse to model the effects of UVR on cutaneous melanomas. Twice weekly exposure to UVR following melanoma initiation did not result in increased tumor formation but did enhance angiotropism and metastasis in some of the UVR-exposed tumors. Sequencing of tumors was not performed, so it is not known whether there were genomic differences between the UVR- and non-UVR-exposed tumors that may have affected metastasis. However, UVR-induced keratinocyte damage led to an increase in Myd88 TLR4+ neutrophils in the UVRexposed mice. Myd88/TLR4 neutrophils enhanced angiotropism of both mouse and human melanoma cells in vivo and in vitro. This study demonstrates that UVR can alter the microenvironment in ways that contribute to tumor progression and metastasis and suggests that UVR on preexisting nevi or early melanomas may contribute to progression.

Using a model of neonatal UVR, Zaidi et al ${ }^{83}$ showed that UVR can recruit interferon-gamma-expressing macrophages that favor melanoma growth and survival. These interferongamma-expressing macrophages were also found in a majority of human melanomas. The role of these macrophages in the development and persistence of melanocytic nevi has yet to be investigated but may be clinically relevant. This response was neonatal specific in the mice tested and may help to explain the significance of childhood sunburn as a risk factor for melanoma.

\section{PERSPECTIVES AND FUTURE DIRECTIONS}

Modeling of human disease begins with defining a specific research question, generating a hypothesis, constructing and testing the model, and finally, relating the results to human disease. ${ }^{84}$ Models are improved through an iterative process of comparing the model with the human disease and improving the model to better replicate elements of the human disease process. By definition, models will not replicate all aspects of human disease and thus these differences need to be carefully identified and considered when applying the results to human patients.

In the case of UVR, melanoma models have primarily focused on two central questions: how does UVR interact directly with melanocytes to induce transformation? and how does UVR modulate the skin microenvironment to facilitate melanoma formation and/or progression? Although UVR is thought to impact melanocytes predominantly, but not exclusively, through mutagenesis, the indirect consequences of UVR are complex and multifaceted, promoting melanoma formation and progression through a variety of mechanisms. Mouse models have been instrumental to advancing our understanding of the many roles of UVR, and emphasis has been placed on effects on the microenvironment and the immune system. However, differences that exist between the mouse and human skin, including the immune system, must be taken into consideration when extrapolating murine findings to humans. Moreover, it is appreciated that, in any particular model, or patient for that matter, the consequences of UVR may vary depending on the circumstances. Ultimately, we anticipate that, with careful planning and thoughtful interpretation of mouse data, further investigation of the role of the microenvironment may reveal biomarkers for progression risk in early melanomas as well as novel preventive or therapeutic targets.

As the field moves forward, there are some important areas where UVR-induced mouse models of melanoma can provide important and novel information. Recent advances in cancer genomics are providing an unpreceded opportunity to connect mouse models and human patients with respect to UVR-induced mutagenesis in melanoma. Comparison of genomic and transcriptomic data from mouse and human melanomas will identify important common and potentially novel genes and/or pathways involved in melanoma genesis and progression. Better understanding of the interaction between mutational landscapes, molecular subtypes, and UVR can provide valuable information concerning cancer prevention, diagnosis, and treatment. Mouse models provide a useful way to investigate how UVR may influence these clinically relevant differences as the dose and frequency of UVR and exposure to other carcinogens can be controlled. There may also be differences in the role of UVR with respect to different cells of origin. ${ }^{85,86}$ Questions about the impact of UVR on cells of origin (eg, melanoblasts $v s$ adult stem cells or junctional $v s$ dermal melanocytes) and the role of the skin microenvironment can be addressed through careful selection 
and manipulation of mouse models. Future work can help elucidate how different doses and timing of UVR can impact the cell of origin for melanoma and the biology and prognosis of the resulting melanomas. Mouse models of UVRassociated melanoma have already provided experimental evidence that UVR can function to induce melanoma and to promote melanoma after oncogene induction and through microenvironmental alterations. As mouse melanoma models continue to be refined, the field will investigate ever more complex and clinically relevant questions about the role of UVR in melanoma.

\section{ACKNOWLEDGMENTS}

This research was supported in part by funding from the intramural research program at the NIH and was also made possible through the NIH Comparative Biomedical Scientist Training Program in partnership with the University of Maryland, College Park and NCl and the NIH Medical Research Scholars Program - a public-private partnership supported jointly by the NIH and generous contributions to the Foundation for the $\mathrm{NIH}$ from the Doris Duke Charitable Foundation, the American Association for Dental Research, the Colgate-Palmolive Company, Genentech, and other private donors. For a complete list, visit the foundation website at http://www.fnih.org.

\section{DISCLOSURE/CONFLICT OF INTEREST}

The authors declare no conflict of interest.

1. Dennis LK, Vanbeek MJ, Beane Freeman LE, et al. Sunburns and risk of cutaneous melanoma: does age matter? A comprehensive metaanalysis. Ann Epidemiol 2008;181:614-627.

2. Ghiasvand R, Weiderpass E, Green AC, et al. Sunscreen use and subsequent melanoma risk: a population-based cohort study. J Clin Oncol 2016;34:3976-3977.

3. Kulichova D, Danova J, Kunte C, et al. Risk factors for malignant melanoma and preventive methods. Cutis 2014;941:241-248.

4. Madigan LM, Lim HW. Tanning beds: impact on health, and recent regulations. Clin Dermatol 2016;34:640-648.

5. Whiteman DC, Whiteman CA, Green AC. Childhood sun exposure as a risk factor for melanoma: a systematic review of epidemiologic studies. Cancer Causes Control 2001;12:69-82.

6. Mitra D, Luo X, Morgan A, et al. An ultraviolet-radiation-independent pathway to melanoma carcinogenesis in the red hair/fair skin background. Nature 2012;491:449-453.

7. Oliveria SA, Saraiya $M$, Geller $A C$, et al. Sun exposure and risk of melanoma. Arch Dis Child 2006;91:131-138.

8. Douki T, Reynaud-Angelin A, Cadet J, et al. Bipyrimidine photoproducts rather than oxidative lesions are the main type of DNA damage involved in the genotoxic effect of solar UVA radiation. Biochemistry 2003;42:9221-9226.

9. El Ghissassi F, Baan R, Straif $K$, et al. A review of human carcinogenspart D: radiation. Lancet Oncol 2009;10:751-752.

10. Wang $S Q$, Setlow $R$, Berwick $M$, et al. Ultraviolet $A$ and melanoma: a review. J Am Acad Dermatol 2001;44:837-846.

11. Kligman LH, Akin FJ, Kligman AM. The contributions of UVA and UVB to connective tissue damage in hairless mice. J Invest Dermatol 1985;84: 272-276.

12. Labat-Robert J, Fourtanier A, Boyer-Lafargue B, et al. Age dependent increase of elastase type protease activity in mouse skin. Effect of UVirradiation. J Photochem Photobiol B 2000;57:113-118.

13. Pearse $A D$, Gaskell $S A$, Marks R. Epidermal changes in human skin following irradiation with either UVB or UVA. J Invest Dermatol 1987;88:83-87.

14. Brash DE. UV signature mutations. Photochem Photobiol 2015;91: $15-26$.

15. Noonan FP, Zaidi MR, Wolnicka-Glubisz A, et al. Melanoma induction by ultraviolet $A$ but not ultraviolet $B$ radiation requires melanin pigment. Nat Commun 2012;3:884.
16. Tyrrell RM. Induction of pyrimidine dimers in bacterial DNA by $365 \mathrm{~nm}$ radiation. Photochem Photobiol 1973;17:69-73.

17. Tyrrell RM, Ley RD, Webb RB. Induction of single-strand breaks (alkalilabile bonds) in bacterial and phage DNA by near UV (365 nm) radiation. Photochem Photobiol 1974;20:395-398.

18. Tsao H, Chin L, Garraway LA, et al. Melanoma: from mutations to medicine. Genes Dev 2012;26:1131-1155.

19. Vidwans SJ, Flaherty $\mathrm{KT}$, Fisher $\mathrm{DE}$, et al. A melanoma molecular disease model. PLoS One 2011;6:e18257.

20. Dong J, Phelps RG, Qiao R, et al. BRAF oncogenic mutations correlate with progression rather than initiation of human melanoma. Cancer Res 2003;63:3883-3885.

21. Pollock PM, Harper UL, Hansen KS, et al. High frequency of BRAF mutations in nevi. Nat Genet 2003;33:19-20.

22. Pollock PM, Meltzer PS. A genome-based strategy uncovers frequent BRAF mutations in melanoma. Cancer Cell 2002;2:5-7.

23. Poynter JN, Elder JT, Fullen DR, et al. BRAF and NRAS mutations in melanoma and melanocytic nevi. Melanoma Res 2006;16:267-273.

24. Uribe P, Wistuba II, Gonzalez S. BRAF mutation: a frequent event in benign, atypical, and malignant melanocytic lesions of the skin. Am J Dermatopathol 2003;25:365-370.

25. Bauer J, Curtin JA, Pinkel D, et al. Congenital melanocytic nevi frequently harbor NRAS mutations but no BRAF mutations. J Invest Dermatol 2007;127:179-182.

26. Karram $S$, Novy $M$, Saroufim $M$, et al. Predictors of BRAF mutation in melanocytic nevi: analysis across regions with different UV radiation exposure. Am J Dermatopathol 2013;35:412-418.

27. Shain AH, Bastian BC. From melanocytes to melanomas. Nat Rev Cancer 2016;16:345-358.

28. Yeh $\mathrm{I}$, von Deimling A, Bastian BC. Clonal BRAF mutations in melanocytic nevi and initiating role of BRAF in melanocytic neoplasia. J Natl Cancer Inst 2013;105:917-919.

29. Wang JX, Fukunaga-Kalabis M, Herlyn M. Crosstalk in skin: melanocytes, keratinocytes, stem cells, and melanoma. J Cell Commun Signal 2016;10:191-196.

30. Haass NK, Herlyn M. Normal human melanocyte homeostasis as a paradigm for understanding melanoma. J Investig Dermatol Symp Proc 2005;10:153-163.

31. Natarajan VT, Ganju P, Ramkumar A, et al. Multifaceted pathways protect human skin from UV radiation. Nat Chem Biol 2014;10l: 542-551.

32. Golan T, Messer AR, Amitai-Lange A, et al. Interactions of melanoma cells with distal keratinocytes trigger metastasis via notch signaling inhibition of MITF. Mol Cell 2015;591:664-676.

33. Hsu MY, Meier FE, Nesbit M, et al. E-cadherin expression in melanoma cells restores keratinocyte-mediated growth control and downregulates expression of invasion-related adhesion receptors. Am J Pathol 2000;156l:1515-1525.

34. Li G, Satyamoorthy K, Herlyn M. N-cadherin-mediated intercellular interactions promote survival and migration of melanoma cells. Cancer Res 2001;611:3819-3825.

35. Li G, Satyamoorthy $\mathrm{K}$, Meier $\mathrm{F}$, et al. Function and regulation of melanoma-stromal fibroblast interactions: when seeds meet soil. Oncogene 2003;221:3162-3171.

36. Chan J, Robinson ES, Atencio J, et al. Characterization of the CDKN2A and ARF genes in UV-induced melanocytic hyperplasias and melanomas of an opossum (Monodelphis domestica). Mol Carcinog 2001;31: $16-26$.

37. Ley RD. Ultraviolet radiation A-induced precursors of cutaneous melanoma in Monodelphis domestica. Cancer Res 1997;57:3682-3684.

38. Nairn RS, Kazianis S, Della Coletta $L$, et al. Genetic analysis of susceptibility to spontaneous and UV-induced carcinogenesis in Xiphophorus hybrid fish. Mar Biotechnol (NY) 2001;3:S24-S36.

39. Robinson ES, VandeBerg JL, Hubbard GB, et al. Malignant melanoma in ultraviolet irradiated laboratory opossums: initiation in suckling young, metastasis in adults, and xenograft behavior in nude mice. Cancer Res 1994;54:5986-5991.

40. Wood SR, Berwick M, Ley RD, et al. UV causation of melanoma in Xiphophorus is dominated by melanin photosensitized oxidant production. Proc Natl Acad Sci USA 2006;103:4111-4115.

41. Jiang W, Ananthaswamy HN, Muller HK, et al. p53 protects against skin cancer induction by UV-B radiation. Oncogene 1999;18: 4247-4253. 
42. Romerdahl CA, Donawho C, Fidler IJ, et al. Effect of ultraviolet-B radiation on the in vivo growth of murine melanoma cells. Cancer Res 1988;48:4007-4010.

43. Epstein JH, Epstein WL, Nakai T. Production of melanomas from DMBAinduced "blue nevi" in hairless mice with ultraviolet light. J Natl Cancer Inst 1967;38:19-30.

44. Husain Z, Pathak MA, Flotte $T$, et al. Role of ultraviolet radiation in the induction of melanocytic tumors in hairless mice following 7, 12-dimethylbenz(a)anthracene application and ultraviolet irradiation. Cancer Res 1991;51:4964-4970.

45. Pathak MA. Ultraviolet radiation and the development of nonmelanoma and melanoma skin cancer: clinical and experimental evidence. Skin Pharmacol 1991;4(Suppl 1):85-94.

46. Ito S, Wakamatsu K. Quantitative analysis of eumelanin and pheomelanin in humans, mice, and other animals: a comparative review. Pigment Cell Res 2003;16:523-531.

47. Thody AJ, Higgins EM, Wakamatsu K, et al. Pheomelanin as well as eumelanin is present in human epidermis. J Invest Dermatol 1991;97: 340-344.

48. Hennessy A, Oh C, Diffey B, et al. Eumelanin and pheomelanin concentrations in human epidermis before and after UVB irradiation. Pigment Cell Res 2005;18:220-223.

49. Eichmuller S, van der Veen C, Moll I, et al. Clusters of perifollicular macrophages in normal murine skin: physiological degeneration of selected hair follicles by programmed organ deletion. J Histochem Cytochem 1998;46:361-370.

50. Pasparakis M, Haase I, Nestle FO. Mechanisms regulating skin immunity and inflammation. Nat Rev Immunol 2014;14:289-301.

51. Birchmeier C, Gherardi E. Developmental roles of HGF/SF and its receptor, the c-Met tyrosine kinase. Trends Cell Biol 1998;8:404-410.

52. Gaggioli C, Deckert M, Robert G, et al. HGF induces fibronectin matrix synthesis in melanoma cells through MAP kinase-dependent signaling pathway and induction of Egr-1. Oncogene 2005;24:1423-1433.

53. Halaban R, Rubin JS, Funasaka $Y$, et al. Met and hepatocyte growth factor/scatter factor signal transduction in normal melanocytes and melanoma cells. Oncogene 1992;7:2195-2206.

54. Straussman R, Morikawa T, Shee K, et al. Tumour micro-environment elicits innate resistance to RAF inhibitors through HGF secretion. Nature 2012;487:500-504.

55. Kos L, Aronzon A, Takayama H, et al. Hepatocyte growth factor/scatter factor-MET signaling in neural crest-derived melanocyte development. Pigment Cell Res 1999;12:13-21.

56. Yamaguchi Y, Hearing VJ. Physiological factors that regulate skin pigmentation. Biofactors 2009;35:193-199.

57. Yang XM, Park M. Expression of the hepatocyte growth factor/scatter factor receptor tyrosine kinase is localized to epithelia in the adult mouse. Lab Invest 1995;73:483-491.

58. Aoki H, Yamada Y, Hara A, et al. Two distinct types of mouse melanocyte: differential signaling requirement for the maintenance of non-cutaneous and dermal versus epidermal melanocytes. Development 2009;136:2511-2521.

59. Imokawa G. Autocrine and paracrine regulation of melanocytes in human skin and in pigmentary disorders. Pigment Cell Res 2004;17:96-110.

60. Noonan FP, Otsuka T, Bang S, et al. Accelerated ultraviolet radiationinduced carcinogenesis in hepatocyte growth factor/scatter factor transgenic mice. Cancer Res 2000;60:3738-3743.

61. Noonan FP, Recio JA, Takayama $\mathrm{H}$, et al. Neonatal sunburn and melanoma in mice. Nature 2001;413:271-272.

62. Gaffal E, Landsberg J, Bald T, et al. Neonatal UVB exposure accelerates melanoma growth and enhances distant metastases in $\mathrm{Hgf}-\mathrm{Cdk} 4$ (R24C) C57BL/6 mice. Int J Cancer 2011;129:285-294.

63. Ha L, Ichikawa $T$, Anver $M$, et al. ARF functions as a melanoma tumor suppressor by inducing p53-independent senescence. Proc Natl Acad Sci USA 2007;104:10968-10973.
64. Thomas J, Liu T, Cotter MA, et al. Melanocyte expression of survivin promotes development and metastasis of UV-induced melanoma in HGF-transgenic mice. Cancer Res 2007;67:5172-5178.

65. Hirobe T. Histochemical survey of the distribution of the epidermal melanoblasts and melanocytes in the mouse during fetal and postnatal periods. Anat Rec 1984;208:589-594.

66. Kelsall SR, Mintz B. Metastatic cutaneous melanoma promoted by ultraviolet radiation in mice with transgene-initiated low melanoma susceptibility. Cancer Res 1998;58:4061-4065.

67. Chin L, Pomerantz J, Polsky D, et al. Cooperative effects of INK4a and ras in melanoma susceptibility in vivo. Genes Dev 1997;11: 2822-2834.

68. Broome Powell M, Gause PR, Hyman P, et al. Induction of melanoma in TPras transgenic mice. Carcinogenesis 1999;20:1747-1753.

69. Kannan K, Sharpless NE, Xu J, et al. Components of the Rb pathway are critical targets of UV mutagenesis in a murine melanoma model. Proc Natl Acad Sci USA 2003;100:1221-1225.

70. Hacker E, Muller HK, Irwin N, et al. Spontaneous and UV radiationinduced multiple metastatic melanomas in Cdk4R24C/R24C/ TPras mice. Cancer Res 2006;66:2946-2952.

71. Viros A, Sanchez-Laorden B, Pedersen $M$, et al. Ultraviolet radiation accelerates BRAF-driven melanomagenesis by targeting TP53. Nature 2014;511:478-482.

72. Luo C, Sheng J, Hu MG, et al. Loss of ARF sensitizes transgenic BRAFV600E mice to UV-induced melanoma via suppression of XPC. Cancer Res 2013;73:4337-4348.

73. De Fabo EC, Noonan FP, Fears T, et al. Ultraviolet B but not ultraviolet A radiation initiates melanoma. Cancer Res 2004;64:6372-6376.

74. Coelho SG, Hearing VJ. UVA tanning is involved in the increased incidence of skin cancers in fair-skinned young women. Pigment Cell Melanoma Res 2010;23:57-63.

75. Hill HZ, Hill GJ. UVA, pheomelanin and the carcinogenesis of melanoma. Pigment Cell Res 2000;13(Suppl 8):140-144.

76. Rouzaud F, Kadekaro AL, Abdel-Malek ZA, et al. MC1R and the response of melanocytes to ultraviolet radiation. Mutat Res 2005;571: 133-152.

77. Wenczl E, Van der Schans GP, Roza L, et al. Pheo)melanin photosensitizes UVA-induced DNA damage in cultured human melanocytes. J Invest Dermatol 1998;111:678-682.

78. Klug HL, Tooze JA, Graff-Cherry C, et al. Sunscreen prevention of melanoma in man and mouse. Pigment Cell Melanoma Res 2010;23: 835-837.

79. Wolf $\mathrm{P}$, Donawho CK, Kripke ML. Effect of sunscreens on UV radiationinduced enhancement of melanoma growth in mice. J Natl Cancer Inst 1994;86:99-105.

80. Loser K, Beissert S. Regulation of cutaneous immunity by the environment: an important role for UV irradiation and vitamin D. Int Immunopharmacol 2009;9:587-589.

81. Byrne SN, Spinks N, Halliday GM. Ultraviolet a irradiation of C57BL/6 mice suppresses systemic contact hypersensitivity or enhances secondary immunity depending on dose. J Invest Dermatol 2002;119: 858-864.

82. Bald T, Quast T, Landsberg J, et al. Ultraviolet-radiation-induced inflammation promotes angiotropism and metastasis in melanoma. Nature 2014;507:109-113.

83. Zaidi MR, Davis S, Noonan FP, et al. Interferon-gamma links ultraviolet radiation to melanomagenesis in mice. Nature 2011;469: 548-553.

84. Thomas RM, Van Dyke T, Merlino G, et al. Concepts in cancer modeling: a brief history. Cancer Res 2016;76:5921-5925.

85. Alexandrov LB, Nik-Zainal S, Wedge DC, et al. Signatures of mutational processes in human cancer. Nature 2013;500:415-421.

86. Martincorena I, Campbell PJ. Somatic mutation in cancer and normal cells. Science 2015:349:1483-1489. 